\title{
Some Problems in the Spectral Theory of Stationary Processes on $R^{d}$
}

\author{
LOREN D. PITT
}

Communicated by the Editors

1. Introduction. We consider the $d$-dimensional Euclidean space $R^{d}$ and a centered, complex-valued, stochastically continuous process $\left\{X_{t}: t \varepsilon R^{d}\right\}$ that is wide-sense stationary in that the covariance function $E X_{t} \bar{X}_{s}$ is assumed to be a function $\rho(t-s)$ of the difference $t-s$. If $S \subset R^{d}$ is any fixed set, then the classical least squares prediction problem for the process $\left\{X_{t}\right\}$ relative to the set $S$ is concerned with minimizing the expected square distance

$$
E\left|X_{\tau}-\sum_{S} a_{s} X_{s}\right|^{2}
$$

where $\tau \notin S$ is fixed and the indicated sum ranges over all possible finite sums with $s \varepsilon S$.

This minimum will not in general be achieved on this space of finite sums but will be uniquely achieved on its closure with respect to the $L^{2}$ norm $\left(E\left|\sum a_{s} X_{s}\right|^{2}\right)^{1 / 2}$. We let $\mathfrak{H C}(S)$ denote this closure. Then any square integrable random variable $Y$ has a unique "best approximation" $\hat{Y}$ in $\mathcal{H C}(S)$. It is desirable to be able to calculate both the "prediction" $\hat{Y}$ of $Y$ and the so called prediction error

$$
E|Y-\hat{Y}|^{2}=\inf E\left|Y-\sum_{S} a_{s} X_{s}\right|^{2}
$$

We now describe a very closely related but distinct problem which in some contexts is more natural. Instead of considering the space $\mathfrak{F}(S)$, we simply expand $S$ into an $\epsilon$-neighborhood, $S_{\epsilon}$, of $S$ and consider the spaces $\mathfrak{H C}\left(S_{\epsilon}\right)$ and their intersection

$$
\overline{\mathfrak{H}}(S)=\bigcap_{\epsilon>0} \mathfrak{H C}\left(S_{\epsilon}\right) .
$$

Again we may consider the prediction problems of calculating the best approximation of $Y$ in $\overline{\mathfrak{H}}(S)$ and of finding the prediction error.

An obvious first step in this direction is to find a useful description of those 
$Y$ for which the prediction error

$$
\sup _{\epsilon>0} \inf E\left|Y-\sum_{S_{e}} a_{s} X_{s}\right|^{2}
$$

equals zero. That is, we seek a description of the space $\overline{\mathfrak{F}}(S)$.

A second related problem concerns the Markov property introduced by McKean in [8]; see also [10] and [11]. Let $\mathcal{S}=\{S\}$ be any collection of open sets and let $\partial S$ denote the boundary of a set in $S .(\partial S)_{\epsilon}$ signifies the $\epsilon$-neighborhood of $\partial S$, and we introduce the spaces $\overline{\mathcal{H}}(\partial S)=\cap \mathcal{H}\left((\partial S)_{\epsilon}\right)$. For $S \boldsymbol{\varepsilon} S$ the "prediction" of $X_{t}$ given $\overline{\mathscr{H}}(S)$ is the unique element $\hat{X}_{t}$ of $\overline{\mathscr{K}}(S)$ with

$$
E\left|X_{t}-\hat{X}_{t}\right|^{2}=\min \left\{E\left|X_{t}-Y\right|^{2}=Y \varepsilon \overline{\mathcal{H}}(S)\right\} .
$$

The process $\left\{X_{t}: t \varepsilon R^{d}\right\}$ will be called Markovian (in the wide sense) relative to $S$ if it satisfies the condition:

$$
X_{t} \varepsilon \overline{\mathfrak{H}}(\partial S) \text { for each } S \varepsilon \mathcal{S} \text { and } t \notin S .
$$

Given $s$, what conditions on the covariance function $\rho(t-s)=E X_{t} \bar{X}_{s}$ will imply that $\left\{X_{t}\right\}$ has this wide sense Markov property relative to $\delta$ ?

Both of these problems have easy translations into problems of weighted trigonometric approximation in several variables. Since $\rho$ is a covariance it must be positive definite. By Bochner's Theorem it follows that $\rho$ has a unique representation

$$
\rho(t)=\int_{R^{d}} e^{i t \cdot x} \Delta(d x),
$$

where $\triangle(d x) \geqq 0$ is a finite Borel measure on $R^{d}$ and $t \cdot x=\sum t_{i} x_{i}$ is the usual inner product on $R^{d}$.

It follows that for any sum

$$
E\left|X_{t}-\sum a_{s} X_{s}\right|^{2}=\int\left|e^{i t \cdot x}-\sum a_{s} e^{i s \cdot x}\right|^{2} \Delta(d x)
$$

and in particular,

$$
E\left|X_{t}-\hat{X}_{t}\right|^{2}=\sup _{\epsilon>0} \inf \int\left|e^{i t \cdot x}-\sum_{S_{e}} a_{s} e^{i s \cdot x}\right|^{2} \Delta(d x) .
$$

Moreover, the map $X_{t} \leftrightarrow e^{i t \cdot x}$ is seen to determine an isometric isomorphism of the space $\mathfrak{F}\left(R^{d}\right)$ onto the Hilbert space $L_{\Delta}^{2}$ consisting of all measurable functions on $R^{d}$ for which

$$
\|f\|^{2}=\|f\|_{\Delta}^{2}=\int|f(x)|^{2} \Delta(d x)<\infty .
$$

Thus, a description of the spaces $\mathfrak{H C}(S)$ and $\overline{\mathcal{C}}(S)$ is completely equivalent to a description of the subspaces $H(S)$ and $\bar{H}(S)$ of $L^{2}{ }_{\Delta}$ where 


$$
\begin{aligned}
H(S) & =\text { closure in } L_{\Delta}^{2} \text { of }\left\{\sum_{S} a_{s} e^{i s \cdot x}\right\} \\
& =\left\{f \varepsilon L_{\Delta}^{2}: \inf \int\left|f(x)-\sum_{S} a_{s} e^{i s \cdot x}\right|^{2} \Delta(d x)=0\right\},
\end{aligned}
$$

and

$$
\tilde{H}(S)=\bigcap_{\epsilon>0} H\left(S_{\epsilon}\right) .
$$

The problem of finding the best approximation $\hat{Y}$ of $Y$ in $\overline{\mathcal{H}}(S)$ is just another description of calculating the orthogonal projection $P$ onto $\bar{H}(S)$ in $L_{\Delta}^{2}$. Similarly for the Markov property we let

$$
\bar{H}(\partial S)=\bigcap_{\epsilon} H\left(\partial S_{\epsilon}\right) .
$$

Then a verification of the Markov property requires that we show $\bar{H}(\partial S)$ contains the range of the restriction of $P$ to $\bar{H}\left(R^{d}-S\right)$.

When $d=1$ and $S$ is an interval these problems have by now acquired fairly satisfactory answers. We state some of the relevant results here, with the simplifying assumption that $\Delta(d x)=\Delta(x) d x$ is absolutely continuous with density $\triangle$ :

If $\int \ln \Delta(x) /\left(1+x^{2}\right)=-\infty$, then $H(0, \infty)=L_{\Delta}^{2}$.

If $\int \ln \Delta(x) /\left(1+x^{2}\right)>-\infty$, then $H(0, \infty)=\mathfrak{T}^{+} \cap L_{\Delta}^{2}$, where $\mathfrak{I}^{+}$

Either

is the Nevanlinna class of analytic functions in the upper half plane. ${ }^{1}$

$$
\begin{aligned}
& \bigcap_{\epsilon>0} H(-T-\epsilon, T+\epsilon)=L_{\Delta}^{2}, \text { or } \\
& \bigcap_{\epsilon>0} H(-T-\epsilon, T+\epsilon)=\varepsilon(T) \bigcap L_{\Delta}^{2},
\end{aligned}
$$

where $\mathcal{E}(T)$ is the space of entire functions of exponential type $\leqq T .{ }^{2}$

If $\int \ln \Delta(x) /\left(1+x^{2}\right)>-\infty$, then $\left\{X_{t}\right\}$ has the Markov property for half (1.6) lines, if and only if, $\triangle^{-1}(x)$ agrees a.e. with the restriction on $R^{1}$ of an entire function of minimal exponential type.

(1.4) goes back to Szego although the formulation in (1.4b) is perhaps new. It may be easily deduced from the Szego alternative (see e.g. [7]) by the methods of [12]. When $\triangle$ is even (1.5) follows from the results stated in [5]. A simple proof may be found in [12]. For (1.6) see [7] and [12].

In this paper we give partial extensions of (1.5) and (1.6) for $d>1$. We note that while these results are new they are not sharp in comparison with the one dimensional results. It should be expected that significant further progress will require different techniques.

We now summarize the contents. Section 2 contains notation and elementary 
material on entire functions of several variables. Sections 3 and 4 discuss the spaces $\bar{H}(s)$ using a comparison technique which relates the spaces $H(s)$ for two measure $\triangle_{1}$ and $\triangle_{2}$ when these measures have approximately the same rates of decay.

More precisely it is shown that if an increasing function $\omega(x)>0$ exists on $x \geqq 0$ for which

$$
\int_{1}^{\infty} \frac{\ln \omega(x)}{x^{2}} d x<\infty
$$

and if for some constant $K$

$$
K^{-1} \omega^{-1}(|x|) \triangle_{1}(d x) \leqq \triangle_{2}(d x) \leqq K \omega(|x|) \triangle_{1}(d x),
$$

then the structure of the family of spaces $H(S)$ is in many ways the same for $\triangle_{1}$ and $\triangle_{2}$. Letting $\Delta_{0}$ be the special weight $\prod_{1}{ }^{d}\left(1+x_{i}{ }^{2}\right)^{-1} d x$, significant information about the spaces $H(S)$ can be obtained for any weight $\triangle$ which satisfies a condition of the form

$$
K^{-1} \omega^{-1}(|x|) \leqq \triangle(x) \leqq K \omega(|x|) .
$$

It is shown, in particular, that if $S \subset R^{d}$ is a bounded symmetric body and if $\triangle$ satisfies (1.9), then $\bar{H}(S)$ consists exactly of those functions $f \varepsilon L_{\Delta}^{2}$ which are restrictions to $R^{d}$ of entire functions on $C^{d}$ which satisfy a certain exponential growth condition.

These conditions are weakened in section 5 but unfortunately the conclusions are also weakened. Thus we can show that if $\int_{R^{d}}(\ln \triangle(x)) \triangle_{0}(x)>-\infty$, then $H(S)$ contains only entire functions but are unable to establish exponential growth estimates without further assumptions on $\triangle$.

In Sections 6 and 7 we discuss the Markov property. We show that if $\left\{X_{t}\right\}$ has the Markov property with respect to the class $S$ of spheres and half spaces then $\Delta^{-1}(x)$ agrees a.e. with an entire function of minimal exponential type. Conversely if $\triangle^{-1}(x)$ is of minimal exponential type and satisfies the growth condition (1.9), then $\left\{X_{t}\right\}$ has the Markov property with respect to the class $\mathcal{S}$ of all open sets.

2. Notations and preliminaries on entire functions. $R^{d}$ denotes the standard Euclidean space of $d$-tuples $t=\left(t_{1}, \cdots, t_{d}\right)$ equipped with the usual inner product $t \cdot x=\sum t_{j} x_{i}$ and norm $|t|=(t \cdot t)^{1 / 2} . C^{d}$ will be the $d$-dimensional complex space of $d$-tuples of complex numbers $z=\left(z_{1}, \cdots, z_{d}\right)$. For $z \varepsilon C^{d}$ we write $z=x+i y$ where $z_{i}=x_{i}+i y_{i}$.

A complex valued function $f(z)$ defined on an open subset of $C^{d}$ is called analytic if it is continuous and analytic in each variable separately. If the domain of an analytic function $f(z)$ is all of $C^{d}$ we say $f(z)$ is entire. If $f(z)$ is entire, it has an everywhere convergent (multiple) power series expansion. As a standard reference on analytic functions of several variables we mention 
the book [2] of Bochner and Martin. The book [14] by Stein and Weiss is also useful for related questions in Fourier analysis.

An entire function $f(z)$ is said to be of exponential type if there are positive constants $M$ and $\gamma$ for which the inequality

$$
|f(z)| \leqq M e^{\gamma|z|}
$$

holds for all $z \varepsilon C^{d}$. The infimum of all permissible $\gamma$ is called the type of $f$.

We will need two modifications of the concept of exponential type. Let $S \subset R^{d}$ be a bounded open convex set satisfying $-S=S$. We will call such an $S$ a symmetric body. For any symmetric body there is an associated dual symmetric body

$$
S^{*}=\left\{y \varepsilon R^{d}:|x \cdot y| \leqq 1 \text { for each } x \varepsilon S\right\},
$$

and an associated norm

$$
\rho(z)=\rho_{S}(z)=\sup _{y \varepsilon S^{*}}|z \cdot y|
$$

defined either on $C^{d}$ or on $R^{d}$.

An entire function $f(z)$ is said to be of exponential type $S$ if for each $\epsilon>0$ there is a constant $M_{\epsilon}$ for which

$$
|f(z)| \leqq M_{\epsilon} e^{(1+\epsilon) \rho(z)}
$$

for all $z \varepsilon C^{d}$. The class of all entire functions of exponential type $S$ is denoted by $\varepsilon(S){ }^{3}$

We note that if $\mu(d t)$ is any finite measure supported on the set $S^{*}$ then,

$$
\left|\int e^{i t \cdot z} \mu(d t)\right| \leqq M e^{\rho(y)},
$$

where $M$ is the total mass of $\mu$ and $y=\operatorname{Im} z$. In particular,

$$
f(z)=\int_{S^{*}} e^{i t \cdot z} \mu(d t)
$$

is an entire function of exponential type $S$.

Another class of functions that we need is the class $\mathcal{E}_{1}(S)$ of entire functions $f(z)$ which for each $\epsilon>0, z \varepsilon C^{d}$ and $a \varepsilon S \subset R^{d}$ satisfy an inequality of the form

$$
|f(z+\lambda a)| \leqq M_{z, a} e^{(1+\epsilon)|\lambda|}
$$

for all $\lambda \varepsilon C^{1}$.

Clearly $\varepsilon_{1}(S) \supset \varepsilon(S)$. Presumably this inclusion is proper.

The classical Paley-Wiener Theorem as proved (though not as stated) by Plancherel and Polya [13] asserts that a square integrable function $f(x)$ is the restriction to $R^{d}$ of an entire function $f(z) \varepsilon \mathcal{E}_{1}(S)$ iff the Fourier transform 


$$
\hat{f}(t)=\int e^{-i t \cdot x} f(x) d x
$$

is supported in $S^{*}$. In particular, $\hat{f}(t)$ is Lebesgue summable, and by Fourier inversion the function $f(z)$ satisfies

$$
|f(z)| \leqq M e^{\rho(y)}
$$

where $y=\operatorname{Im} z$. Thus $f \varepsilon \varepsilon(S)$ and

$$
\varepsilon_{1}(S) \cap L^{2}=\varepsilon(S) \cap L^{2} .
$$

This combination of results will simply be referred to as the Paley-Wiener theorem in the sequel.

An elementary consequence of the Paley-Wiener theorem is that for any $\phi$ of exponential type $<\epsilon$ which is bounded on $R^{d}$ and for any $f \varepsilon L^{2} \cup L^{1}$, the support of the Fourier transform of $\phi \cdot f$ is contained in an $\epsilon$-neighborhood of the support of the Fourier transform of $f$.

We close this section with several needed facts about functions of exponential type. The first two are obvious.

If $f \varepsilon \varepsilon(S)$ and $g \varepsilon \varepsilon(T)$, then $f \cdot g \varepsilon \varepsilon((1 / 2)(T \cap S))$.

For each fixed symmetric body $S$ and each positive $\alpha<1$ there is an $\epsilon>0$ so that $\phi f \varepsilon \varepsilon(\alpha S)$ whenever $f \varepsilon \varepsilon(S)$ and $\phi$ is of type $<\epsilon$.

The following lemma for functions of one variable is useful.

Lemma 2.1. ([6], p. 21). Let $f(z)$ be analytic in the circle $|z| \leqq 2 e R$ with $f(0)=1$ and $|f(z)| \leqq M$ for $|z| \leqq 2 e R$. Let $\eta>0$ be an arbitrary number $<(3 e) / 2$. Then inside the circle $|z| \leqq R$, but outside of a family of excluded circles the sum of whose radii is not greater than $4 \eta R$, we have

$$
\ln |f(z)|>-\left(2+\ln \frac{3 e}{2 \eta}\right) \ln M .
$$

By choosing $\eta=\epsilon / 10$ and making very rough estimates the lemma easily yields the following:

Lemma 2.2. Let $f(z)$ be an entire function of the single complex variable $z$ with $f(0)=1$ which satisfies the growth condition $|f(z)|<M e^{T|z|}$. Then for each $R>0$ and $0<\epsilon<1 / 2$ there is an $r$ with $(1-\epsilon) R<r<R$ so that for $|z|=r$

$$
|f(z)|>M^{-H(\epsilon)} e^{-K(\epsilon) T|z|}
$$

for

$$
H(\epsilon)=6-\ln \epsilon
$$

and

$$
K(\epsilon)=68-20 \ln \epsilon .
$$

From this lemma we easily obtain: 
Lemma 2.3. If $f, g$ and $h=g / f$ are entire functions of the single complex variable $z$ satisfying $f(0)=1,|f(z)| \leqq M e^{T|z|}$ and $|g(z)|<N e^{S|z|}$, then for each $\epsilon>0$,

$$
|h(z)|<N M^{H(\epsilon)} e^{(1 /(1-\epsilon))[S+K(\epsilon) T]|z|} .
$$

In particular $h(z)$ is of exponential type.

The analogue of the inequality (2.7) also holds in several variables. In fact, suppose $f$ is a symmetric body and $f$ and $g$ are entire functions which satisfy

$$
f(0)=1,|f(z)| \leqq M e^{\rho(z)} \text { and }|g(z)| \leqq N e^{\rho(z)} .
$$

Then for each $z \varepsilon C^{d}$ with $\rho(z)=1$ and each $\lambda_{\varepsilon} C^{1}$ we have

$$
|f(\lambda z)| \leqq M e^{|\lambda|} \text { and }|g(\lambda z)| \leqq N e^{|\lambda|}
$$

If we assume in addition that $h(z)=g(z) / f(z)$ is entire, then

$$
\sup _{\rho(z) \leqq r}|h(z)|=\sup _{\rho(z)=1} \sup _{|\lambda| \leqq r}\left|\frac{g(\lambda z)}{f(\lambda z)}\right|,
$$

and (2.7) now supplies

$$
N M^{H(\epsilon)} e^{(1 /(1-\epsilon))[1+K(\epsilon)]|r|}
$$

as an upper bound for the right hand side of (2.8).

We summarize this discussion with the following

Lemma 2.4. (a) If $f$ and $g$ are of exponential type and $h=g / f$ is entire, then $h$ is of exponential type.

(b) Let $h$ be an entire function and let $S$ be a symmetric body. If for each $\epsilon>0$ we can find a function $f$ of exponential type $<\epsilon$ and such that hf $\varepsilon \mathcal{E}((1-\epsilon) S)$, then $h \varepsilon \varepsilon(S)$.

In the sequel we are mainly concerned with a positive weight $\Delta(d x)$ and the subspaces $H(S)$ and $\bar{H}(S)$ of $L^{2}{ }_{\Delta}$ introduced in Section 1. The following elementary lemma will be used repeatedly.

Lemma 2.5. Let $S$ be a symmetric body and suppose $f \varepsilon \varepsilon(S)$ is bounded on $R^{d}$. Then $f \varepsilon H\left(S^{*}\right)$.

Proof. Let $\psi_{\epsilon}(x)=\prod_{1}^{d}\left(\sin \left(\epsilon x_{j}\right) / \epsilon x_{j}\right)$. Then $f \psi_{\epsilon}$ is of exponential type and $f \psi_{\epsilon} \varepsilon L^{2}$. Choose $\delta>0$ and let $\left(S^{*}\right)_{\delta}$ be a $\delta$-neighborhood of $S^{*}$. By the Paley-Wiener theorem we may choose $\epsilon$ so small that the Fourier transform of $f \psi_{\epsilon}$ is supported on $\left(S^{*}\right)_{\delta}$. By Fourier inversion $f \psi_{\epsilon}$ may be approximated in $L^{2}$ by sums of the form

$$
\sum_{\left(S^{*}\right)_{28}} a_{s} e^{i s \cdot x} .
$$

Hence $f \psi_{\epsilon} \varepsilon H\left(\left(S^{*}\right)_{2 \delta}\right)$ and letting $\epsilon \rightarrow 0$ we see that $f \varepsilon H\left(\left(S^{*}\right)_{2 \delta}\right)$ for each $\delta>0$. From this we have $f \varepsilon \bar{H}\left(S^{*}\right)$. 
But for $f \varepsilon \mathcal{E}(S)$ and $0<\alpha<1$ we have $f_{\alpha}(x) \equiv f(\alpha x) \varepsilon \mathcal{E}((1 / \alpha) S)$. Since $f$ is bounded, we have by the above reasoning that $f_{\alpha} \varepsilon \bar{H}(\alpha S) \subset H(S)$. Letting $\alpha \rightarrow 1$ we see that $f=\lim _{\alpha \rightarrow 1} f_{\alpha} \varepsilon H(S)$.

3. A special weight. Some of the main results in later sections are obtained by comparison of $L_{\Delta}^{2}$ norms for different weights $\triangle(d x)$. In order that this work, we need something to compare with. We choose the special density

$$
\Delta_{0}(x)=\prod_{1}^{d}\left(1+x_{i}^{2}\right)^{-1} .
$$

We now establish a number of properties for $\triangle_{0}$ that we shall use later. If $f \varepsilon L_{\Delta_{0}}^{2}$, then $f_{\epsilon}(x)=f(x) \prod_{1}^{d}\left(\sin \epsilon x_{i}\right) /\left(\epsilon x_{j}\right) \varepsilon L^{2} \cap L_{\Delta_{\circ}}^{2}$ and as $\epsilon \rightarrow 0$, $f_{\epsilon} \rightarrow f$ in $L_{\Delta_{0}}^{2}$. Further, if $S$ is a symmetric body, if $f \varepsilon \varepsilon\left(S^{*}\right)$, and if $0<\alpha<1$, we may choose $\epsilon$ so small that $f_{\epsilon} \varepsilon \varepsilon\left(\alpha S^{*}\right)$. Thus $f_{\epsilon}$ is bounded and by Lemma 2.5, $f \varepsilon \bar{H}\left(\alpha^{-1} S\right)$. Letting $\epsilon \downarrow 0$ and then letting $\alpha \uparrow 1$ we conclude that $\bar{f} \varepsilon \bar{H}(S)$ and thus $\varepsilon\left(S^{*}\right) \cap L_{\Delta 。}^{2} \subset \bar{H}(S)$.

For $0<\alpha<1$ the following inequalities hold;

$$
\triangle_{0}(\alpha x) \geqq \triangle_{0}(x) \geqq \alpha^{2 d} \triangle_{0}(\alpha x) .
$$

Thus the transformation $f(x) \rightarrow f(\alpha x)$ is a bounded operator on $L_{\Delta 。}^{2}$ and as $\alpha \rightarrow 1, f(\alpha x) \rightarrow f(x)$ in $L_{\Delta_{0}}^{2}$. Further, if $f \varepsilon \bar{H}(S)$, then $f(\alpha x) \varepsilon \bar{H}(\alpha S) \subset H(S)$ so that $f(x)=\lim _{\alpha \rightarrow} 1 f(\alpha x) \varepsilon H(S)$ and we have

$$
H(S)=\bar{H}(S) \text {. }
$$

Next we observe that if $f \varepsilon H(S)$, there is a sequence $f_{n}(x)=\sum_{S} a_{n, s} e^{i s \cdot x}$ converging to $f$ in $L_{\Delta 。}^{2}$. The sequence

$$
f_{n, \epsilon}(x)=f_{n}(x) \cdot \prod_{1}^{d} \frac{\sin \epsilon x_{i}}{\epsilon x_{i}}
$$

then converges to $f_{\epsilon}(x)=f(x) \prod_{1}{ }^{d}\left[\sin \epsilon x_{i} / \epsilon x_{i}\right]$ in $L^{2}$. Moreover, $f_{n, \epsilon} \varepsilon \&\left(S^{*}{ }_{d \epsilon}\right)$ so that $f_{\epsilon} \varepsilon \mathcal{E}\left(S^{*}{ }_{d \epsilon}\right)$. By Lemma 2.4 we may conclude that $f \varepsilon \varepsilon\left(S^{*}\right)$ and that $H(S) \subset \mathcal{E}\left(S^{*}\right)$.

Combining the above remarks we have:

Lemma 3.1. If $S$ is a symmetric body, then

$$
H(S)=\bar{H}(S)=\varepsilon\left(S^{*}\right) \cap L_{\Delta 。}^{2} .
$$

Comment. We mention here that the equality of the two spaces $H(S)$ and $\bar{H}(S)$ is for a general weight a very delicate question. At present only the most elementary facts are known. The proof that $H(S)=\bar{H}(S)$ which we just gave for $\triangle_{0}$ works with minor modifications if $\Delta(x)$ is radially monotonic or almost so. Counterexamples with rather irregular $\Delta$ can be produced in dimension 1.

One further fact is known and of interest. For any two numbers $\alpha$ and $\beta$ 
with $0<\alpha<\beta$ we have $H(\alpha S) \subseteq \tilde{H}(\alpha S) \subseteq H(\beta S)$. Because $L^{2}{ }_{\Delta}$ is separable the inequality $H(\alpha S) \neq \bar{H}(\alpha S)$ can occur for at most countable many $\alpha$.

If the set $S \subset R^{d}$ is only assumed to be measurable we have:

Lemma 3.2. Let $S \subset R^{d}$ and suppose $f \varepsilon L_{\Delta_{0}}^{2} \cap L^{2}$ is such that

$$
0=\int e^{-i t \cdot x} f(x) d x
$$

a.e. on $R^{d}-S$; then $f \varepsilon \bar{H}(S)$.

Proof. Since $f \varepsilon L^{2}$ we may approximate $f$ in $L^{2}$ and a fortiori in $L_{\Delta 。}^{2}$ with functions $f_{n}$ for which the functions $\int e^{-i t \cdot x} f_{n}(x) d x$ have compact support contained in an $\epsilon$-neighborhood $S_{\epsilon}$ of $S$. As in the proof of Lemma 2.5 these approximating functions are in $\bar{H}\left(S_{\epsilon}\right)$ and hence $f \varepsilon \bigcap_{\epsilon} \bar{H}\left(S_{\epsilon}\right)=\bar{H}(S)$.

Lastly we will need:

Lemma 3.3. Let $S$ and $T$ be closed subsets of $R^{d}$ such that for each $\epsilon>0$ a $\delta>0$ can be found with

$$
S_{\delta} \cap T_{\delta} \subset(S \cap T)_{\epsilon}
$$

then

$$
\bar{H}(S) \cap \bar{H}(T)=\bar{H}(S \cap T){ }^{4}
$$

Proof. Clearly $\bar{H}(S \cap T) \subset \bar{H}(S) \cap \bar{H}(T)$. To obtain the opposite inclusion it suffices by Lemma 3.2 to show that for each $\epsilon>0$ and $f \varepsilon \bar{H}(S) \cap \bar{H}(T)$ we may approximate $f$ in $L_{\Delta \text { 。 }}^{2}$ by functions $f_{\alpha}$ in $L^{2}$ which satisfy

$$
0=\int e^{-i t \cdot x} f_{\alpha}(x) d x \text { for } t \varepsilon R^{d}-(S \cap T)_{\epsilon} .
$$

But $f_{\alpha}(x)=f(x) \prod_{1}^{d}\left(\sin \alpha x_{i} / \alpha x_{j}\right)(\alpha \downarrow 0)$ is just such an approximating family. In fact, if we choose $\delta$ as in (3.3) and then take $\alpha$ small enough it is clear that (3.5) is satisfied.

4. Related measures. It will be shown that a pair of equivalence relations exist among weights which preserve much of the essential structure of the spaces $\bar{H}(S) \subset L_{\Delta}^{2}$.

Definition 4.1. Let $\triangle_{1}$ and $\triangle_{2}$ be weights on $R^{d}$. For $S \subset R^{d}$ we let $\bar{H}_{i}(S) \subset$ $L^{2}{ }_{\Delta_{i}}(i=1,2)$ denote the appropriate spaces. We say $\triangle_{1}$ and $\triangle_{2}$ are related if there exists a sequence $\phi_{n}$ of entire functions on $C^{d}$ and constants $K_{n}$ which satisfy
a) $\phi_{n}$ is of exponential type $n^{-1}$,
b) the $\phi_{n}$ are non-negative and uniformly bounded on $R^{d}$,
c) $\lim \phi_{n}(x)=1$ for all $x \varepsilon R^{d}$, and
d) $\phi_{n}(x) \triangle_{1}(d x) \leqq K_{n} \triangle_{2}(d x)$,
$\phi_{n}(x) \triangle_{2}(d x) \leqq K_{n} \triangle_{2}(d x)$. 
We say $\triangle_{1}$ and $\triangle_{2}$ are weakly related if a sequence $\phi_{n}$ exists which satisfies conditions a), b) and c) and

$\left.\mathrm{d}^{\prime}\right)$ for each compact set $S$ the multiplication operator $f(x) \rightarrow \phi_{n}(x) f(x)$ is bounded from $\bar{H}_{1}(S)$ into $L_{\Delta_{2}}^{2}$ and from $\bar{H}_{2}(S)$ into $L_{\Delta_{1}}^{2}$.

We note that if $\Delta_{1}$ and $\Delta_{2}$ are related, then they are weakly related. Also observe that

$$
\phi_{n} \bar{H}_{1}(S) \subset \bar{H}_{2}\left(S_{\epsilon}\right) \text { and } \phi_{n} \bar{H}_{2}(S) \subset \bar{H}_{1}\left(S_{\epsilon}\right) \text { for } \epsilon>n^{-1} .
$$

Examples of equivalent weights are not difficult to find but after excluding such elementary examples as rational weights they are not especially cheap either. We will make significant use of the following result that may be found in $([4]$, p. 146):

Lemma 4.2. Let $\omega(x)>0$ be a continuous and eventually increasing function on $[0, \infty)$ satisfying

$$
\int_{0}^{\infty} \frac{\ln \omega(x)}{1+x^{2}} d x<\infty ;
$$

then there is an even entire $\varphi(x)$ of exponential type 1 so that $\varphi(0)=1, \varphi(x) \geqq 0$ and $\varphi(x) \omega(x)$ is bounded.

This lemma is used to prove:

Lemma 4.3. Suppose $\triangle_{1}(d x)$ and $\triangle_{2}(d x)$ are two weights satisfying an inequality of the form

$$
c^{-1} \omega^{-1}(|x|) \triangle_{1}(d x)<\triangle_{2}(d x)<c \omega(|x|) \triangle_{1}(d x),
$$

where $\omega(x)>0$ is an increasing function satisfying (4.1). Then $\Delta_{1}$ and $\Delta_{2}$ are related.

Proof. Let $T(x)=\ln \omega(x)$ and let $\lambda_{n}>0$ be constants with $\sum \lambda_{n} T(n x)<\infty$ for all $x>0$ and for which $\sum n \lambda_{n}<\infty$. For example we may choose $\lambda_{n}=$ $\left(n^{3} T\left(n^{2}\right)\right)^{-1}$. It follows that $\sum \lambda_{n} \int_{(0, \infty)} T(n x)\left(1+x^{2}\right)^{-1} d x<\infty$ and there exists an $f(x)>0$ and increasing to $+\infty$ for which $S(x)=f(x) \sum \lambda_{n} T(n x)$ satisfies

$$
\int_{0}^{\infty} \frac{S(x) d x}{1+x^{2}}<\infty
$$

Let $W(x)=\exp \{S(x)\}$. Then $W(x)$ satisfies the conditions of Lemma 4.2 and for each $\alpha>0$

$$
\lim _{x \rightarrow \infty} \omega(\alpha x) / W(x)=0 .
$$

Now let $\varphi(z)$ be the function satisfying $\varphi(x) W(x)<K<\infty$ whose existence is guaranteed by Lemma 4.2. Since $\varphi(z)$ is even it has the form $\varphi(z)=f\left(z^{2}\right)$ and we may produce the desired sequence of functions $\phi_{n}$ by the recipe 


$$
\begin{aligned}
\phi_{n}(z) & =\phi_{n}\left(z_{1}, \cdots, z_{d}\right) \\
& =f\left(\left(z_{1}{ }^{2}+\cdots+z_{d}{ }^{2}\right) / n^{2}\right) .
\end{aligned}
$$

$\left\{\phi_{n}\right\}$ obviously satisfies conditions a), b) and c) of definition 4.1. Moreover,

$$
\begin{aligned}
\sup _{x \in R^{d}} \phi_{n}(x) \omega(|x|) & =\sup \varphi(|x| / n) \omega(|x|) \\
& =\sup \varphi(|x|) \omega(n|x|)
\end{aligned}
$$

and by (4.3) this is finite. Together with condition (4.2) this completes the proof of the lemma.

Comment. As an example, any weight $\triangle(x)$ with

$$
c^{-1} e^{-|x| / \ln ^{2}|x|} \leqq \Delta(x) \leqq c e^{|x| / \ln ^{2}|x|}
$$

is related to the weight $\triangle_{0}$ of Section 3 .

The utility of this relation is illustrated in the next lemma. $\bar{H}_{1}(S)$ and $\bar{H}_{2}(S)$ denote the appropriate subspaces of $L_{\Delta_{1}}^{2}$ and $L_{\Delta_{2}}^{2}$ as defined in (1.2).

Lemma 4.4. Let $\triangle_{1}$ and $\triangle_{2}$ be related weights. Each of the following properties for $\triangle_{1}$ implies the corresponding property for $\triangle_{2}$.

i). For each closed set $S \subset R^{d}, \bar{H}_{1}(S)$ contains each $f \varepsilon L^{2} \cap L_{\Delta_{1}}^{2}$ for which $\int e^{-i t \cdot x} f(x) d x$ vanishes on $R^{d}-S$.

ii). The relation $\bar{H}_{1}(S) \cap \bar{H}_{1}(T)=\bar{H}_{1}(S \cap T)$ holds for each pair of closed sets $S$ and $T$ for which, when $\epsilon>0$ is given, there exists $a \delta$ with

$$
\left(S_{\delta} \cap T_{\delta}\right) \subset(S \cap T)_{\epsilon} .
$$

iii). $\quad \mathcal{E}\left(S^{*}\right) \cap L_{\Delta_{1}}^{2} \subset \bar{H}_{1}(S)$ for each symmetric body $S \subset R^{d}$.

iv). $\quad \bar{H}_{1}(S) \subset \mathcal{E}\left(S^{*}\right)$ for each symmetric body $S \subset R^{d}$.

Proof. Let $\left\{\phi_{n}\right\}$ be a sequence satisfying a), b), c) and d) in Definition 4.4. i). Let $f \varepsilon L_{\Delta_{2}}^{2} \cap L^{2}$ be such that $\int e^{-i t \cdot x} f(x)=0$ on $R^{d}-S$. For $\epsilon>0$ we choose $n>\epsilon^{-1}$ with $\int\left|f-\phi_{n}{ }^{2} f\right|^{2} \triangle_{2}(d x)<\epsilon$. Setting $\phi=\phi_{n}$ it suffices to show

$$
0=\inf \int\left|\phi^{2} f-\sum_{S_{2 e}} a_{s} e^{i s \cdot x}\right|^{2} \Delta_{2}(d x) .
$$

But $\phi H_{2}\left(S_{\epsilon}\right) \subset H_{2}\left(S_{2 \epsilon}\right)$ so it also suffices to show

$$
0=\inf \int\left|\phi^{2} f-\phi \sum_{S_{e}} a_{s} e^{i s \cdot x}\right|^{2} \triangle_{2}(d x) .
$$

Now $\phi$ is bounded by some constant $M$ and $\phi \triangle_{2}(d x) \leqq K \triangle_{1}(d x)$ so that

$$
\begin{aligned}
\inf \int\left|\phi^{2} f-\phi \sum_{S_{e}} a_{s} e^{i s \cdot x}\right|^{2} \triangle_{2}(d x) & \\
& \leqq K M \text { inf } \int\left|\phi f-\sum_{S_{e}} a_{s} e^{i s \cdot x}\right|^{2} \triangle_{1}(d x)
\end{aligned}
$$


However, $\phi f \varepsilon L^{2} \cap L_{\Delta_{1}}^{2}$ and $\int e^{-i t \cdot x} \phi(x) f(x)$ vanishes on $R^{d}-S_{\epsilon}$, so by assumption the term on the right of (4.5) is zero, and (4.3) follows.

ii). Let $f \varepsilon \bar{H}_{2}(S) \cap \bar{H}_{2}(T)$, let $\epsilon>0$ be given and choose $\delta>0$ with $S_{\delta} \cap T_{\delta} \subset$ $(S \cap T)_{\epsilon}$. Now pick $n>2 \delta^{-1}>2 \epsilon^{-1}$ with

$$
\int\left|f-\phi^{2} f\right|^{2} \triangle_{2}(d x)<\epsilon
$$

where $\phi=\phi_{n}$. Proceeding as in part (i) it will suffice to show

$$
0=\inf \int\left|\phi^{2} f-\phi \sum_{(S \cap T)_{2} e} a_{s} e^{i s \cdot x}\right|^{2} \triangle_{2}(d x) .
$$

But the term on the right is bounded by a constant times

$$
\inf \int\left|\phi f-\sum_{(S \cap T)_{2} e} a_{s} e^{i s \cdot x}\right|^{2} \Delta_{1}(d x) .
$$

Moreover, $\phi f \varepsilon H_{1}\left(S_{\delta}\right) \cap H_{1}\left(T_{\delta}\right) \subset \bar{H}_{1}\left(S_{\epsilon} \cap T_{\epsilon}\right)$. Thus (4.6) vanishes and the proof of (ii) is complete.

iii). Let $f \varepsilon \varepsilon\left(S^{*}\right) \cap L_{\Delta_{2}}^{2}$ and let $\epsilon>0$ be fixed. Choose $n>\epsilon^{-1}$ with $\int\left|f-\phi^{2} f\right|^{2} \triangle_{2}(d x)<\epsilon$. We need to show

$$
0=\inf \int\left|\phi^{2} f-\phi \sum_{S_{2}} a_{s} e^{i s \cdot x}\right|^{2} \Delta_{2}(d x)
$$

But,

$$
\begin{aligned}
\inf \int\left|\phi^{2} f-\phi \sum_{S_{2 e}} a_{s} e^{i s \cdot x}\right|^{2} \triangle_{2}(d x) & \\
& \leqq \text { const. inf } \int\left|\phi f-\sum_{S_{2} e} a_{s} e^{i s \cdot x}\right|^{2} \triangle_{1}(d x) \\
& =0
\end{aligned}
$$

since $\phi f \varepsilon L_{\Delta_{1}}^{2} \cap \mathcal{E}\left(\left(S_{\epsilon}\right)^{*}\right)$.

iv). Assuming $\bar{H}_{1}(S) \subset \varepsilon\left(S^{*}\right)$ and $f \varepsilon \bar{H}_{2}(S)$ it follows that $f \cdot \phi_{n}{ }^{2} \varepsilon \bar{H}_{1}\left(S_{2 \epsilon}\right) \subset$ $\mathcal{E}\left(\left(S_{2 \epsilon}\right)^{*}\right)$ provided $\epsilon>n^{-1}$. Lemma 2.4 implies $f \varepsilon \varepsilon\left(S^{*}\right)$.

Remark. The proofs of (i), (ii) and (iv) given above are valid when $\triangle_{1}$ and $\triangle_{2}$ are weakly related provided $S$ is bounded. The proof of (iii) is also valid in this setting if we also have $\phi_{n} f \varepsilon L_{\Delta_{1}}^{2}$ whenever $f \varepsilon \varepsilon\left(S^{*}\right) \cap L_{\Delta_{2}}^{2}$. In what follows we exhibit a class of weights that are weakly related to the weight $\triangle_{0}$ of Section 3 and which satisfy this added condition.

We let $A$ be any measurable set of finite measure and we write $1_{B}(x)$ for the indicator function of the set $B=R^{d}-A$. Set $\triangle_{2}(d x)=1_{B}(x) d x$. I claim that on the space $L^{2} \cap \mathcal{E}\left(S^{*}\right)$ the two measures $d x$ and $\triangle_{2}(d x)$ yield equivalent $L^{2}$ norms.

To see this we let $K=\left\{f \varepsilon L^{2}\right.$ : support $\left.f \subset A\right\}$ and $L=\varepsilon\left(S^{*}\right) \cap L^{2}$. It 
suffices to show that the subspaces $K$ and $L$ of $L^{2}$ have a positive angle between them.

Bringing in the orthogonal projections $P$ and $Q$ onto the spaces $K$ and $L$, it suffices to show that the operator norm of the self-adjoint operator $P Q P$ is less than 1.

If we use Fourier transforms to express $P Q P$ as an integral operator we find

$$
(P Q P) f(x)=\int k(x, y) f(y) d y
$$

where

$$
k(x, y)=\text { const. } 1_{A}(x) 1_{A}(y) \int_{S} e^{i s \cdot(y-x)} d s .
$$

Clearly, $k$ is a Hilbert-Schmidt kernel and $P Q P$ is compact. The norm of $P Q P$ thus equals its maximum eigenvalue $\lambda_{0} \leqq 1$. But $\lambda_{0} \neq 1$ since any $f \varepsilon L$ is analytic and thus cannot be supported on the set $A$.

With this result the following proposition is elementary.

Lemma 4.5. Let $A \subset R^{d}$ satisfy

$$
\int_{A} e^{T|x|} d x<\infty \text { for all } T>0
$$

and let $\omega(x)>0$ be an increasing function on $(0, \infty)$ with

$$
\int_{1}^{\infty} \frac{\ln \omega(x)}{x^{2}} d x<\infty .
$$

Each weight $\triangle(d x)$ satisfying an inequality of the form

$$
1_{R^{d}-A}(x) d x<K \omega(|x|) \triangle(d x)
$$

is weakly related to the weight $\triangle_{0}$ by a sequence $\phi_{n}(z)$ for which the multiplication operators

$$
\phi_{n}: \mathcal{E}\left(S^{*}\right) \cap L_{\Delta}^{2} \rightarrow L_{\Delta 。}^{2}
$$

are bounded for each symmetric body $S \subset R^{d}$.

Proof. Let $\left\{\phi_{n}(z)\right\}$ be the sequence constructed in the proof of Lemma 4.3. Because $\phi_{n}(x) \omega(|x|)$ is bounded (4.10) implies $\triangle(d x) \geqq$ const. $\phi_{n}(x) d x$ when-, ever $x \varepsilon R^{d}-A$. Since $\left\{\phi_{n}\right\}$ satisfies conditions a), b) and c) of Definition (4.1) conditions $d^{\prime}$ ) of Definition (4.1) and (4.11) are all that need be verified.

Let $S$ be a symmetric body and $f \varepsilon \bar{H}_{0}(S)=\varepsilon\left(S^{*}\right) \cap L_{\Delta_{0}}^{2}$. Then $\phi_{n} f \varepsilon L^{2} \cap$ $\mathcal{E}\left(\left(S_{\epsilon}\right)^{*}\right)$ and for some constant $C=C(S)$ independent of $f$ we have

$$
\left\|\phi_{n} f\right\|_{\infty}^{2} \leqq C \int\left|\phi_{n} f\right|^{2} d x
$$

and 


$$
\begin{aligned}
\int\left|\phi_{n} f\right|^{2} \Delta_{1}(d x) & \leqq C\left|\int \Delta_{1}(d x)\right| \int\left|\phi_{n} f\right|^{2} d x \\
& \leqq \text { const. } \int|f|^{2} \Delta_{0}(d x) .
\end{aligned}
$$

Thus $\phi_{n}: H_{0}(S) \rightarrow L^{2}$ is bounded.

To prove (4.11) let $f \varepsilon \varepsilon\left(S^{*}\right) \cap L_{\Delta}^{2}$. Then

$$
\begin{aligned}
\int\left|\phi_{n} f\right|^{2} \Delta_{0}(d x) & \leqq \int\left|\phi_{n} f\right|^{2} d x \\
& \leqq \int_{A}\left|\phi_{n} f\right|^{2} d x+\int_{R^{d}-A}\left|\phi_{n} f\right|^{2} d x
\end{aligned}
$$

Now $\int_{A}\left|\phi_{n} f\right|^{2} d x$ is finite by (4.8) since $\phi_{n} f$ is of exponential type, and $\int_{R^{d-A}}\left|\phi_{n} f\right|^{2} d x$ is finite by (4.10).

Thus $\int\left|\phi_{n} f\right|^{2} d x<\infty$ and there is a constant independent of $f$ for which

$$
\begin{aligned}
\int\left|\phi_{n} f\right|^{2} d x & \leqq C \int_{R^{d}-A}\left|f \phi_{n}\right|^{2} d x \\
& \leqq \text { const. } \int_{R^{d}}|f|^{2} \Delta(d x)
\end{aligned}
$$

This proves (4.11). In turn this implies $\bar{H}(S) \subset \mathcal{E}\left(S^{*}\right)$ and that $\phi_{n}: \bar{H}(S) \rightarrow L_{\Delta \text { 。 }}^{2}$ is bounded which completes the proof. Together with Lemma 4.4 and the subsequent remark this yields:

Proposition 4.6. Suppose $\omega(x)>0$ is increasing and satisfies (4.9). Suppose also that $\triangle(d x)$ satisfies

$$
K \omega(|x|) \triangle(d x) \geqq d x,
$$

except on a set $A$ with $\int_{A} e^{T|x|} d x<\infty$, for all $T>0$. Then

a) If $S$ is a symmetric body, then $\bar{H}(S)=\varepsilon\left(S^{*}\right) \cap L^{2}$.

b) If $S \subset R^{d}$ is compact and $f \varepsilon L_{\Delta}^{2} \cap L^{2}$ is such that $\int e^{-i t \cdot x} f(x)$ vanishes on $R^{d}-S$, then $f \varepsilon \bar{H}(S)$.

c) If $S$ is compact and $T$ is closed, then

$$
\bar{H}(S) \cap \bar{H}(T)=\bar{H}(S \cap T) .
$$

5. Conditions which imply $H(S) \subset \varepsilon\left(\mathbf{S}^{*}\right)$. Lemma 4.4 and Proposition 4.6 give conditions on $\triangle$ which imply $\bar{H}(S)=\mathcal{E}\left(S^{*}\right) \cap L^{2}{ }_{\Delta}$ for each convex body $S$. In this section we pursue the question of the inclusion $\bar{H}(S) \subset \varepsilon\left(S^{*}\right)$ further. We begin by stating a useful lemma of de Branges (see [3] or [12]).

Lemma 5.1. Let $\triangle(d x)$ be a finite weight on $R^{1}$ and let $g \varepsilon L_{\Delta}{ }_{\Delta}$ satisfy

$$
0=\int e^{i t x} g(x) \Delta(d x), \text { whenever }|t| \leqq T .
$$


If $f(z)$ is an entire function of exponential type $T$ and is bounded on $R^{1}$, then

$$
0=\int f(x) g(x) \Delta(d x)
$$

In several dimensions we decompose the weight $\triangle(d x)$ on $R^{d}$ into the marginal distribution $d \omega\left(x_{2}, \cdots, x_{d}\right)$ of the variables $x_{2}, \cdots, x_{d}$ and the conditional distribution $k\left(x_{2}, \cdots, x_{d} ; d x_{1}\right)$ of the variable $x_{1}$ when $x_{2}, \cdots, x_{d}$ are given. That is, $\triangle(d x)=d \omega\left(x_{2}, \cdots, x_{d}\right) k\left(x_{2}, \cdots, x_{d} ; d x_{1}\right)$. Lemma 5.1 now yields:

Lemma 5.2. Let $\Delta(d x)=d \omega\left(x_{2}, \cdots, x_{d}\right) k\left(x_{2}, \cdots, x_{d} ; d x_{1}\right)$ be a finite weight on $R^{d}$. Let $g(x) \varepsilon L^{1}{ }_{\Delta}$ satisfy

$$
0=\int e^{i t \cdot x} g(x) \Delta(d x) \text { whenever }\left|t_{1}\right| \leqq T .
$$

If $f(x)$ is bounded and if for each choice of $x_{2}, \cdots, x_{d}$ the function $f\left(\xi, x_{2}, \cdots, x_{d}\right)$ is entire and of exponential type $T$ in $\xi$, then

$$
\mathbf{0}=\int f(x) g(x) \Delta(d x) .
$$

Proof. If $\left|t_{1}\right| \leqq T$,

$$
\begin{aligned}
\mathbf{0} & =\int e^{i t \cdot x} g(x) \Delta(d x) \\
& =\int_{R^{d-1}} d \omega\left(x_{2}, \cdots, x_{d}\right) e^{i} \sum_{2}^{d} t_{j} x_{i}\left\{\int e^{i t_{1} x_{1}} g\left(x_{1}, \cdots, x_{d}\right) k\left(x_{2}, \cdots, x_{d} ; d x_{1}\right)\right\}
\end{aligned}
$$

Since this is true for arbitrary $t_{2}, \cdots, t_{d}$ we may conclude for a.a. $[\omega]$ choices of $x_{2}, \cdots, x_{d}$ that

$$
0=\int e^{i t_{1} x_{1}} g\left(x_{1}, \cdots, x_{d}\right) k\left(x_{2}, \cdots, x_{d} ; d x_{1}\right) \quad \text { if } \quad\left|t_{1}\right|<T .
$$

Applying Lemma 5.1 we have

$0=\int f\left(x_{1}, \cdots, x_{d}\right) g\left(x_{1}, \cdots, x_{d}\right) k\left(x_{2}, \cdots, x_{d} ; d x_{1}\right)$ for a.a. $x_{2}, \cdots, x_{d}$, and

$$
\begin{aligned}
\mathbf{0} & =\int_{R^{d-1}} d \omega\left(x_{2}, \cdots, x_{d}\right)\left\{\int_{R} f\left(x_{1}, \cdots, x_{d}\right) g\left(x_{1}, \cdots, x_{d}\right) k\left(x_{2}, \cdots, x_{d} ; d x_{1}\right)\right\} \\
& =\int f(x) g(x) \Delta(d x) .
\end{aligned}
$$

For fixed positive numbers $T_{1}, \cdots, T_{d}$ we now introduce the pair of open sets 


$$
\begin{aligned}
& S=\bigcap_{i}\left\{t \varepsilon R^{d}:\left|t_{i}\right|<T_{i}\right\} \\
& \tilde{S}=\bigcup_{i}\left\{t \varepsilon R^{d}:\left|t_{i}\right|<T_{i}\right\} .
\end{aligned}
$$

Lemma 5.3. Let $\triangle(d x)$ be a finite weight on $R^{d}$ and let $S$ and $\tilde{S}$ be defined as in (5.1). Let $g \varepsilon L^{1}{ }_{\Delta}$ be such that for some $\epsilon>0$,

$$
0=\int e^{i t \cdot x} g(x) \Delta(d x)
$$

for all $t \varepsilon \widetilde{S_{\epsilon}}$. Then for any $f$ of the form

$$
\begin{gathered}
f(z)=\sum_{S} a_{s} e^{i s \cdot z} \\
0=\int \frac{f(z)-f(\xi)}{\prod\left(z_{j}-\xi_{j}\right)} g(\xi) \Delta(d \xi)
\end{gathered}
$$

for each $z$ with $\prod_{1}^{d} \operatorname{Im} z_{i}=\prod_{1}^{d} y_{i} \neq 0$.

Proof. For each $z=x+i y$ with $\prod y_{i} \neq 0$ we write

$$
\frac{f(z)-f(\xi)}{\prod\left(z_{i}-\xi_{i}\right)}=\sum f_{i}(\xi)
$$

where

$$
\begin{aligned}
f_{i}(\xi)=\prod_{k \neq j}\left(z_{k}-\xi_{k}\right)^{-1} & \\
& \frac{\left.f\left(z_{1}, \cdots, z_{j}, \xi_{i+1}, \cdots, \xi_{j}\right)-f\left(z_{1}, \cdots, z_{i-1}, \xi_{i}, \cdots, \xi_{d}\right)\right\}}{z_{j}-\xi_{i}} .
\end{aligned}
$$

For fixed $\xi_{j+1}, \cdots, \xi_{d}$ the function $f_{i}(\xi)$ is of exponential type $T_{i}$ in the variable $\xi_{i}$. Applying Lemma 5.7 to the variable $\xi_{i}$ we see that $0=\int f_{j}(\xi) g(\xi) \triangle(d \xi)$ and the proof follows.

Proposition 5.4. If $\bar{H}(\widetilde{S}) \neq L_{\Delta}^{2}$, then $\bar{H}(S)$ contains only entire functions.

Proof. For convenience we assume that $\int \Delta(d x)=1$. We then choose an $\epsilon>0$ for which $H\left(\widetilde{S}_{\epsilon}\right) \neq L_{\Delta}^{2}$ and a $g \varepsilon L^{2}{ }_{\Delta}$ with $\|g\|_{\Delta}=1$ and satisfying

$$
\int e^{i t \cdot \xi} g(\xi) \Delta(d \xi)=0 \text { for } t \varepsilon \tilde{S}_{\epsilon}
$$

For each $z \varepsilon C^{d}$ we set

$$
L(z)=\sup \left\{|f(z)|:|| f \|_{\Delta} \leqq 1 \text { and } f(z)=\sum_{S_{\odot}} a_{s} e^{i s \cdot x}\right\}
$$

Clearly $1 \leqq L(z)$, and $L(z)$ is lower semi-continuous and subharmonic. We will show that $L(z)$ is in fact bounded on compact sets. Just as in the one variable case this will imply that $\left\{f=\sum_{S_{e}} a_{s} e^{i s \cdot x}:\|f\|_{\Delta} \leqq 1\right\}$ is a normal family and that $\vec{H}(S) \subset H\left(S_{\epsilon}\right)$ contains only entire functions. 
Proceeding, we use Lemma 5.3 to conclude that for each $f(z)=\sum_{S_{e}} a_{s} e^{i_{s} \cdot z}$ we have

$$
f(z) \int \frac{g(\xi) \Delta(d \xi)}{\prod\left(z_{i}-\xi_{i}\right)}=\int \frac{f(\xi) g(\xi)}{\prod\left(z_{i}-\xi_{i}\right)} \Delta(d \xi) \text { if } \quad \prod y_{i} \neq 0 .
$$

Setting $h(z)=\int g(\xi)\left[\Pi\left(z_{i}-\xi_{i}\right)\right]^{-1} \Delta(d \xi)$ this gives

$$
|f(z)||h(z)| \leqq \int|f(\xi) g(\xi)| \Delta(d \xi) \cdot \frac{1}{\prod y_{i}}
$$

and

$$
L(z)|h(z)| \leqq \frac{1}{\prod y_{i}}
$$

We observe that $h\left(z_{1}+i, \cdots, z_{d}+i\right)$ is bounded and analytic in the domain $\left\{z: y_{i}>0 ; i=1, \cdots, d\right\}$. It follows from the Poisson-Jensen inequality that

$$
\int\left|\frac{\ln \mid h\left(x_{1}+i, \cdots, x_{d}+i\right)}{\prod\left(1+x_{i}^{2}\right)}\right|<\infty .
$$

Since $L(z) \geqq 1$ we have $\ln L(z) \geqq 0$ and when combined with (5.6) and (5.7) this implies

$$
0 \leqq \int \frac{\ln L\left(x_{1}+i, \cdots, x_{d}+i\right)}{\prod\left(1+x_{i}^{2}\right)}<\infty .
$$

Similarly we can show that for any choice $\epsilon_{1}, \cdots, \epsilon_{d}$ of the signs + and - we have

$$
0 \leqq \int \frac{\ln L\left(x_{1}+\epsilon_{1} i, \cdots, x_{d}+\epsilon_{d} i\right)}{\prod\left(1+x_{j}^{2}\right)}<\infty .
$$

Now let $f$ be of the form $f(z)=\sum_{S_{e}} e^{i s \cdot z}$ and observe that

$$
F(z)=e^{i \sum\left(T_{j}+\epsilon\right)\left(z_{j}-i\right)} f(z)
$$

$\mathrm{i}_{\mathrm{S}}$ bounded on the domain $\left\{z: y_{i}>-1 ; j=1, \cdots, d\right\}$.

The Poisson-Jensen inequality applied to $F$ gives

$$
\begin{aligned}
& \ln |f(z)| \leqq \sum\left(T_{i}+\epsilon\right)\left(y_{i}+1\right) \\
&+\frac{\prod_{1}^{d}\left(y_{i}+1\right)}{\pi^{d}} \int \frac{\ln \left|f\left(\xi_{1}-i, \cdots, \xi_{d}-i\right)\right|}{\prod_{1}^{d}\left(\left(y_{i}+1\right)^{2}+\left(x_{i}-\xi_{j}\right)^{2}\right)} \\
& \text { for } y_{i}>-1 ; j=1, \ldots, d .
\end{aligned}
$$

Together (5.9) and (5.10) imply that $L(z)$ satisfies 


$$
\begin{aligned}
& \ln L(z) \leqq \sum_{1}^{d}\left(T_{i}+\epsilon\right)\left(y_{i}+1\right) \\
&+\frac{\prod_{1}^{d}\left(y_{j}+1\right)}{\pi^{d}} \int \frac{\ln L\left(\xi_{1}-i, \cdots, \xi_{d}-i\right)}{\prod_{1}^{d}\left(\left(y_{i}+1\right)^{2}+\left(x_{i}-\xi_{i}\right)^{2}\right)}<\infty \\
& \text { if } y_{1}, \cdots, y_{i}>-1 .
\end{aligned}
$$

(5.11) implies $L(z)$ is locally bounded on the domain $\left\{z: y_{1}, \cdots, y_{d}>-1\right\}$. Similar results hold in the other domains of the form $\left\{z: \epsilon_{1} y_{1}>-1, \cdots\right.$, $\left.\epsilon_{d} y_{d}>-1\right\}$ for each choice $\epsilon_{1}, \cdots, \epsilon_{d}$ of + and - signs. As the union of these domains covers $C^{d}$ it follows that $L(z)$ is locally bounded.

Comment 1. If $\triangle(d x)=\Delta(x) d x$ satisfies $\int\left(\ln \Delta(x) / \prod\left(1+x_{j}{ }^{2}\right)\right)>-\infty$, then the Poisson-Jensen inequality applied to the sector $\left\{z: y_{1}, \cdots, y_{d}>0\right\}$ implies that

$$
\int \frac{\ln L\left(x_{1}+i, \cdots, x_{d}+i\right)}{\prod\left(1+x_{i}^{2}\right)}<\infty
$$

and the above proof needs no essential modifications to show that $\vec{H}(S)$ contains only entire functions for each compact set $S$.

Since invertible real linear changes of variables preserve both the class of entire functions and the class of compact sets we may state:

Proposition 5.5. Let $\triangle$ be a weight on $R^{d}$ which is absolutely continuous and suppose that for some choice $\xi_{1}, \cdots, \xi_{d}$ of linear coordinate variables

$$
\int \frac{\ln \Delta(\xi)}{\prod\left(1+\xi_{j}^{2}\right)}>-\infty
$$

is satisfied. Then for any compact set $\bar{H}(S)$ contains only entire functions.

Comment 2. Let $t \varepsilon R^{d}$ with $|t|=1$ and fix $\epsilon>0$. Define

$$
S_{t, \epsilon}=\{s: t \cdot s>\epsilon|s|\} \text {. }
$$

By a non-singular linear change of variables we may assume

$$
S_{t, \epsilon} \subset\left\{S: S_{1}, \cdots, S_{d}>0\right\} .
$$

If $\tilde{H}\left(R^{d}-S_{t, \epsilon}\right) \neq L_{\Delta}^{2}$ there will exist a nonzero $g \varepsilon L^{2}{ }_{\Delta}$ with

$$
0=\int e^{i S \cdot x} g(x) \Delta(d x) \text { unless } S_{1}, \cdots, S_{d}>0 .
$$

Setting

$$
g_{T}(x)=e^{-i T\left(x_{1}+\cdots+x_{d}\right)} g(x)
$$


we see that

$$
0=\int e^{i S \cdot x} g_{T}(x) \Delta(d x)=0 \text { unless } S_{1}, \cdots, S_{d}>T .
$$

As a result of Proposition 5.4 we may then conclude that the following proposition is true.

Proposition 5.6. If for some $t \varepsilon R^{d}$ with $|t|=1$ and for some $\epsilon>0, \bar{H}\left(R^{d}-\right.$ $\left.S_{t, \epsilon}\right) \neq L_{\Delta}^{2}$, then for each compact set $S \subset R^{d}, \bar{H}(S)$ contains only entire functions.

Comment 3. When $d=1$, the inequality (5.11) and the Phragmen-Lindelöf principle easily combine to give $L(z)=O\left(e^{(T+\epsilon)|z|}\right)$ and thus $\bar{H}(S) \subset \mathcal{E}\left(S^{*}\right)$. For $d>1$ the relation $L(z)=O\left(e^{M|z|}\right)$ for some $M<\infty$ does not follow from (5.11). We can however conclude from the one variable result that for fixed $z_{2}, \cdots, z_{d}$ and $0<|\theta|<\pi$

$$
\lim _{R \rightarrow \infty} \frac{L\left(R \mathrm{e}^{i \theta}, z_{2}, \cdots, z_{d}\right)}{R \sin \theta}=T_{1} .
$$

Together with Phragmen-Lindelöf this shows for each $f \varepsilon \bar{H}(S)$ that $f\left(\xi, z_{2}, \cdots, z_{d}\right)$ is of exponential type $T_{1}$ in $\xi$.

This comment, when applied separately to each possible choice of orthogonal variables in $R^{d}$ gives:

Proposition 5.7. Let $\triangle$ be a weight on $R^{d}$ and suppose that for each choice $x_{1}, \cdots, x_{d}$ of orthogonal coordinate variables the following conditions are true:

a) The absolutely continuous part $\omega(x) d x$ of $\triangle(d x)$ satisfies

$$
\int \frac{\ln \omega(x)}{\Pi\left(1+x_{i}{ }^{2}\right)}>-\infty .
$$

b) For each $T>0$ and $\widetilde{S}=\bigcup_{i}\left\{t:\left|t_{j}\right|<T\right\}$,

$$
\bar{H}(\widetilde{S}) \neq L_{\Delta}^{2} \text {. }
$$

Then for each symmetric body $S \subset R^{d}$,

$$
\bar{H}(S) \subset \mathcal{E}_{1}\left(S^{*}\right) .
$$

We mention the obvious gap between this result and part a) of Proposition 4.6 which holds for weights which are weakly related to the weight $\triangle_{0}$ of Section 3. Not only have we not shown here that $\bar{H}(S) \subset \mathcal{E}\left(S^{*}\right)$ but we also lack any part of the inclusion $\varepsilon\left(S^{*}\right) \cap L^{2}{ }_{\Delta} \subset \bar{H}(S)$. Only in very special cases such as when $\triangle(d x)$ is a radial measure and $S$ is a sphere have we been able to supply these missing details.

6. A necessary condition for $\left\{\mathrm{X}_{t}\right\}$ to be Markovian. Let $\left\{X_{t}: t \varepsilon R^{d}\right\}$ have spectral measure $\triangle$. For each $\epsilon>0$ let $B_{\epsilon} \subset R^{d}$ be the ball of radius $\epsilon>0$ centered at the origin. Define 


$$
\mathcal{H}_{\infty}=\bigcap_{\epsilon} H\left(R^{d}-B_{\epsilon}\right) .
$$

For a pair $K \subseteq L$ of Hilbert spaces $L \ominus K$ will denote the orthogonal complement of $K$ in $L$.

In Sections 8 and 9 of [11] the following facts were established:

I. If $\mathfrak{H}_{\infty}=\{0\}$, then $\triangle$ is absolutely continuous with a density $\triangle(x)$ satisfying $\Delta(x)>0$ a.e., and if $\Delta$ is absolutely continuous, then either $\mathfrak{H}_{\infty}=$ $\{0\}$ or $\mathfrak{H}_{\infty}=\mathfrak{H C}\left(R^{d}\right)$.

II. If for some $\epsilon, \mathfrak{H C}\left(B_{\epsilon}\right) \cap\left\{\mathfrak{H}\left(R^{d}\right) \ominus \mathfrak{H}\left(R^{d}-B_{\epsilon}\right)\right\} \neq\{0\}$, then $\triangle(x)$ has the form $\Delta(x)=f(x) / g(x)$ where $f$ and $g$ are entire functions of exponential type $2 \epsilon$.

III. If $\left\{X_{t}\right\}$ has the Markov property with respect to the collection of all half spaces, then for each $y \varepsilon R^{d}$ the function $\Delta^{-1}(x+\lambda y)$ is entire in $\lambda \varepsilon C^{1}$ for a.a. $x \varepsilon R^{d}$.

By I, for any process with density $\triangle$ either $\mathcal{F}_{\infty}=\{0\}$ or $\mathcal{F}_{\infty}=\mathfrak{F C}\left(R^{d}\right)$. The alternative $\mathfrak{H}_{\infty}=\mathfrak{F}\left(R^{d}\right)$ implies $\mathfrak{H}\left(B_{\epsilon}\right) \subset \mathfrak{H C}\left(R^{d}\right)=\mathfrak{F}\left(R^{d}-B_{\epsilon}\right)$ for each $\epsilon$, and if $\left\{X_{t}\right\}$ is assumed Markovian with respect to spheres this implies $\mathfrak{H C}\left(B_{\epsilon}\right) \subset$ $\mathfrak{F}\left(\partial B_{\epsilon}\right)$. From our viewpoint of Markov processes this looks quite degenerate and we feel justified in restricting our attention to processes which satisfy

$$
\mathfrak{H}\left(\partial B_{\epsilon}\right) \neq \mathfrak{H}\left(B_{\epsilon}\right) \text { for each } \epsilon>0 .
$$

Assuming the Markov property we then have

$$
\{0\} \neq \mathfrak{F}\left(B_{\epsilon}\right) \ominus \mathfrak{H}\left(\partial B_{\epsilon}\right) \subset \mathfrak{F}\left(B_{\epsilon}\right) \cap\left\{\mathfrak{H}\left(R^{d}\right) \ominus \mathfrak{H C}\left(R^{d}-B_{\epsilon}\right)\right\} .
$$

By II, $\triangle^{-1}$ is the ratio $f / g$ of two entire functions that may be chosen of arbitrary small exponential type. We will now prove:

Lemma 6.1. If $h(z)$ is the ratio of two entire functions and if for $y \varepsilon R^{d}$ the function $h(x+\lambda y)$ is an entire function of $\lambda_{\varepsilon} C^{1}$ for a.a. $x \varepsilon R^{d}$, then $h(z)$ is entire.

Together with the preceding comments and Lemma 2.4 this implies:

Proposition 6.2. Let $\left\{X_{t}\right\}$ have the spectral density $\triangle(x)$. Assume $\left\{X_{t}\right\}$ is Markovian with respect to all half spaces and that condition (6.1) holds. Then $\triangle^{-1}(x)$ is entire of minimal exponential type.

The proof of Lemma 6.1 will be reduced to the case $d=2$. For this we need the preliminary:

Lemma 6.3. Assume the conditions of Lemma 6.1 are satisfied and that $x_{0}$ and $y_{0}$ are such that $g\left(x_{0}+\lambda y_{0}\right)$ is not identically zero. Then $h$ is analytic at each point $\xi_{0}$ of the form $\xi_{0}=x_{0}+\lambda_{0} y_{0}$.

Proof. Since $h=f / g$ we only need to consider the case when $g\left(\xi_{0}\right)=0$. By Rouché's theorem there are positive numbers $\epsilon$ and $\epsilon^{\prime}$ for which $g(x+\lambda y) \neq 0$ whenever $\left|\lambda-\lambda_{0}\right|=\epsilon$ and $\left|z-z_{0}\right|<\epsilon^{\prime}$. If, in addition $z$ is such that $h(x+\lambda y)$ is entire we have 


$$
h(x+\lambda y)=\frac{1}{2 \pi i} \int_{\left|\xi-\lambda_{0}\right|=\epsilon} \frac{h(x+\xi y)}{\xi-\lambda} d \xi .
$$

For any $z$ with $\left|z-z_{0}\right|<\epsilon^{\prime}, h(x+\lambda y)$ is a meromorphic function of $\lambda$. Also the right side of (6.2) is continuous in $z$ and analytic in $\lambda$. For $\left|\lambda-\lambda_{0}\right|<\epsilon$ the equation (6.2) is valid for a dense set of $z$ with $\left|z-z_{0}\right|<\epsilon^{\prime}$ and we may conclude that (6.2) is valid for all $\lambda$ and $z$ with $\left|\lambda-\lambda_{0}\right|<\epsilon$ and $\left|z-z_{0}\right|<\epsilon^{\prime}$.

Now as a function of $x$ the right side of (6.2) is analytic near $x_{0}$ and we may conclude that $h$ is analytic at $\xi_{0}=x_{0}+\lambda_{0} y_{0}$.

Note that for each $x_{0} \varepsilon R^{d}$ there is a $y_{0}$ for which $g\left(x_{0}+\lambda y_{0}\right)$ is not identically zero and hence $h$ is analytic at $x_{0}$.

Proof of Lemma 6.1. Let $x_{0} \varepsilon R^{d}$ and note the condition that $h(x+\lambda y)=$ $f(x+\lambda y) / g(x+\lambda y)$ is entire for a.a. fixed $x$ justifies the simplifying assumption that $g$ does not vanish identically an any $d-1$ dimensional affine surface of $R^{d}$ which contains $x_{0}$. When $d=2$ this and Lemma 6.3 complete the proof.

When $d>2$ we note that Lemma 6.3 implies that the restriction of $h$ to any such hyperplane must also satisfy the conditions of Lemma 6.1. Proceeding by induction we may conclude that whenever the other variables are held fixed $h\left(z_{1}, \cdots, z_{d}\right)$ is entire and $z_{k}$. By Hartog's theorem we conclude that $h$ is entire.

7. A sufficient Condition that $\left\{\mathbf{X}_{t}\right\}$ be Markovian. Let $\left\{\mathbf{X}_{t}\right\}$ be given with the spectral measure $\triangle(d x)$ and let $\delta=\{S\}$ be a class of sets in $R^{d}$. In order to show that $\left\{X_{t}\right\}$ has the Markov property with respect to the class $S$ it suffices to show for each $S \varepsilon \&$ and $t \notin S$ that the projection $g(x)$ of $e^{i t \cdot x}$ onto $\bar{H}(S)$ is in $\bar{H}(\partial S)$. We can reduce this problem further by showing first that $g$ \& $\bar{H}(S) \cap \bar{H}\left(R^{d}-S\right)$ and then prove that $\bar{H}(S) \cap \bar{H}\left(R^{d}-S\right)=\bar{H}(\partial S)$. Moreover, we note that $g \varepsilon \bar{H}(S) \cap \bar{H}\left(R^{d}-S\right)$ iff $h(x)=e^{i t \cdot x}-g(x)$ is in $\bar{H}\left(R^{d}-S\right)$ since $g \varepsilon \bar{H}(S)$ and $e^{i t \cdot x} \varepsilon \bar{H}\left(R^{d}-S\right)$. Observing further that $h$ satisfies $0=\int e^{-i s \cdot x} h(x) \triangle(d x)$ for all $s \varepsilon S$ we may state the next lemma.

Lemma 7.1. $\left\{X_{t}\right\}$ is Markovian with respect to $S$ if for each $S \mathbf{\varepsilon} S$,

i) $\bar{H}(S) \cap \bar{H}\left(R^{d}-S\right)=\bar{H}(\partial S)$ and

ii) for $h \varepsilon L_{\triangle}^{2}$ the relation

$$
0=\int e^{-i s \cdot x} h(x) \triangle(d x) \quad \text { for all } \quad s \varepsilon S
$$

implies $h \varepsilon \bar{H}\left(R^{d}-S\right)$.

Theorem 7.2. Let $\left\{X_{t}\right\}$ have the spectral density $\triangle(x)$, and suppose thal $\triangle^{-1}(x)$ agrees almost everywhere with the restriction to $R^{d}$ of a function of minimat exponential type. Suppose also that $\delta=\{S\}$ is the class of all open sets and that $\triangle$ is related to the weight $\triangle_{0}(x)$. Then $\left\{X_{t}\right\}$ is Markovian with respect to $\mathrm{S}$.

With elementary modifications of the proof we may also show:

Theorem 7.3. Let $\triangle^{-1}(x)$ be of minimal exponential type and suppose there 
is an increasing $\omega(x)>0$ satisfying (4.9) and

$$
\triangle^{-1}(x)<K \omega(|x|)
$$

except on a set $A$ satisfying

$$
\int_{A} e^{T|x|} d x<\infty \quad \text { for each } T>0 .
$$

Then $\left\{X_{t}\right\}$ is Markovian with respect to the class $\mathcal{S}$ of all bounded open sets.

We shall only prove Theorem 7.2 as Theorem 7.3 does not involve anything essentially different.

We begin by noting that our conditions together with Lemmas 4.4, 4.3 and 3.3 imply that condition (i) of Lemma 7.1 is satisfied. ${ }^{5}$ Thus we only need show that for each $h \varepsilon L^{2}$ which satisfies

$$
0=\int e^{-i s \cdot x} h(x) \Delta(x) d x
$$

for all $s \varepsilon S$ we have $h \varepsilon \bar{H}\left(R^{d}-S\right)$.

Since $\triangle$ is related to $\triangle_{0}$ we can choose the relating sequence $\phi_{n}$ with

$$
\phi_{n}(x) \triangle(x) \leqq K_{n} \text { and } \phi_{n} \leqq K_{n} \triangle(x)
$$

for some choice of constants $K_{n}$.

We observe that $\phi_{n} h \rightarrow h$ in $L_{\Delta}^{2}$ and hence it suffices to show that if $\epsilon>0$ is fixed and $n>\epsilon^{-1}$, then $\phi_{n} h \varepsilon \tilde{H}\left(\left(R^{d}-S\right)_{\epsilon}\right)$. By Lemmas (3.2) and (4.4) it suffices to show $\phi_{n} h \varepsilon L^{2}$ and that

$$
\widehat{\phi_{n}} h(s)=\int e^{-i s \cdot x} \phi_{n}(x) h(x) d x
$$

is supported on $\left(\overline{R^{d}-S}\right)$. That $\phi_{n} h \varepsilon L^{2}$ follows from (7.4) since

$$
\begin{aligned}
\int\left|\phi_{n} h\right|^{2} d x & \leqq \text { const. } \int\left|h^{2} \phi_{n}\right| \triangle(x) d x \\
& \leqq \text { const. } \int\left|h^{2}\right| \triangle(x) d x .
\end{aligned}
$$

That the support of $\widehat{\phi_{n} h}$ is contained in $\left(R^{d}-S\right)_{\epsilon}$ follows from the PaleyWiener Theorem since

$$
\widehat{\phi_{n} h}(s)=\int e^{-i s \cdot x}(h(x) \Delta(x))\left(\phi_{n}(x) \Delta^{-1}(x)\right) d x
$$

and $\phi_{n} \triangle^{-1}$ is an entire function of exponential type $n^{-1}$ that is bounded on $R^{d}$. This completes the proof.

\section{Footnotes}

1. An analytic function $f(z)$ on $\operatorname{Im} z>0$ is in $\mathfrak{T}^{+}$iff $f=\mathrm{B} \cdot 0$ where $B$ is bounded and $O(z)$ satisfies $\ln |O(x+i y)|=(y / \pi) \mid=(y / \pi) \int\left(\ln |O(C)| /(x-C)^{2}+y^{2}\right) d C$. That is, 0 is outer. 
2. An entire function $f(z)$ is of exponential type $T \geq 0$ if for each $\epsilon>0,|f(z)|=O\left(e^{(T+\epsilon)|z|}\right)$. If $f(z)$ is of type $T=0$ we say it is of minimal exponential type. Note that for some discrete measures it may happen that $L^{2}{ }_{\Delta}=\mathcal{E}(T) \cap L^{2}{ }_{\Delta}$.

3. Note that $\varepsilon(S) \subset \varepsilon(T)$ if $T \subset S$.

4. Condition (3.3) holds if $S$ or $T$ is compact.

5. Observe $S_{\epsilon} \cap\left(R^{d}-S\right)_{\epsilon}=(\partial S)_{\epsilon}$.

\section{Note}

After this paper was submitted I received from Professor S. Kotani of Kyoto University an interesting preprint, On a Markov property for stationary Gaussian processes with a multidimensional parameter, which contains an alternative method for obtaining the essential results of Section 6 and 7 in the present paper.

\section{REFERENCES}

1. A. Beurling \& P. Malliavan On Fourier transforms of measures with compact support, Acta Math. 107 (1962). 291-309.

2. S. Bochner \& W. T. Martin Several complex variables, Princeton, 1948.

3. L. DE Branges, Local operators on Fourier transforms, Duke Math. J. 25 (1958), 143-153.

4. P. Koosis, Sur la non-totalité de certaines suites d'exponentielles, Ann. Sci. l'École Norm. Sup. (3) 75 (1958), 125-152.

5. M. G. KReIN, On a fundamental approximation problem in the theory of extrapolation and filtration of stationary random processes, Dokl. Akad. Nauk SSSR 94 (1964), 13-16; Selected Transl. in Math. Stat. and Prob., Vol. 4, Providence, 1963, 127-132.

6. B. Ja. Levin, Distribution of zeros of entire functions, Amer. Math. Soc. Transl. of Monographs 5, Providence, 1964.

7. N. Levinson \& H. P. McKean JR., Weighted trigonometrical approximation on $R^{1}$ with applications to the germ field of a stationary Gaussian noise, Acta Math. 112 (1964), 99-143.

8. H. P. McKean Jr., Brownian motion with a several dimensional time, Theory Prob. Applications 8 (1963), 335-354.

9. G. M. Molchan, On some problems concerning Brownian motion in Levy's sense, Theory Prob. Applications 12 (1967), 682-690.

10. G. M. Molchan, Characterization of Gaussian fields with Markovian property, Soviet Math. Dokle 12 (1971), 563-567.

11. L. D. Piтt, A Markov property for Gaussian processes with a multidimensional parameter, Arch. Rat. Mech. Anal. 43 (1971), 367-391.

12. L. D. Pitr, On problems of trigonometrical approximation from the theory of stationary Gaussian processes, Jour. Multivariate Analysis 2 (1972), 145-161.

13. M. Plancherel \& G. Pólya, Fonctions entires et intégrales de Fourier multiples, Commentarii Math. Helvetici 9 (1937), 224-248.

14. E. M. Stein \& G. Weiss, Introduction to Fourier analysis on Euclidean spaces, Princeton, 1971.

This research was partially supported by Army Research Office Grant ARO-D-1005.

University of Virginia

and

University of Southern California, Los Angeles

Date communicated: JANUARY 30, 1973 